\title{
A Transcriptional Sequencing Analysis of Islet Stellate Cell and Pancreatic Stellate Cell
}

\author{
Xiaohang Wang, ${ }^{1}$ Wei Li, ${ }^{1}$ Juan Chen, ${ }^{1}$ Sheng Zhao, ${ }^{2}$ Shanhu Qiu, ${ }^{1}$ Han Yin, \\ Vladmir Carvalho, ${ }^{1}$ Yunting Zhou, ${ }^{1}$ Ruifeng Shi ${ }^{D},{ }^{1}$ Jiannan Hu, ${ }^{1}$ Shenyi Li, ${ }^{1}$ \\ Munire Nijiati, ${ }^{1}$ and Zilin Sun (D) ${ }^{1}$ \\ ${ }^{1}$ Department of Endocrinology, Institute of Diabetes, School of Medicine, Zhongda Hospital, Southeast University, Nanjing, China \\ ${ }^{2}$ Department of Biochemistry and Molecular Biology, School of Medicine, Southeast University, Nanjing, China
}

Correspondence should be addressed to Zilin Sun; sunzilin1963@126.com

Received 12 September 2017; Accepted 22 November 2017; Published 24 January 2018

Academic Editor: Hiroshi Okamoto

Copyright (c) 2018 Xiaohang Wang et al. This is an open access article distributed under the Creative Commons Attribution License, which permits unrestricted use, distribution, and reproduction in any medium, provided the original work is properly cited.

\begin{abstract}
Background. Our previous studies have shown that islet stellate cell (ISC), similar to pancreatic stellate cell (PSC) in phenotype and biological characters, may be responsible for the islet fibrosis in type 2 diabetes. To further identify the differences between PSC and ISC and for better understanding of the physiological function of ISC, we employed genome-wide transcriptional analysis on the PSCs and ISCs of Wistar rats. Method. PSCs and ISCs from each rat were primarily cultured at the same condition. Genome-wide transcriptional sequence of stellate cells was generated. The identified differentially expressed genes were validated using RT-PCR. Results. 32 significant differentially expressed genes between PSCs and ISCs were identified. Moreover, collagen type 11a1 (COL11A1), was found to be expressed 2.91-fold higher in ISCs compared with PSCs, indicating that COL11A1 might be a potential key gene modulating the differences between PSC and ISC. Conclusions. Our study identified and validated the differences between PSC and ISC in genome-wide transcriptional scale, confirming the assumption that ISC and PSC are similar other than identical. Moreover, our data might be instrumental for further investigation of ISC and islet fibrosis, and some differential expressed genes may provide an insight into new therapeutic targets for type 2 diabetes.
\end{abstract}

\section{Introduction}

Type 2 diabetes mellitus (T2DM) is a chronic disorder which is characterized by $\beta$-cell dysfunction and insulin resistance $[1,2]$. As the prevalence of type 2 diabetes continues to increase, it is imperative to seek a better understanding of its pathogenesis and find more efficient treatments to decrease the morbidity and ease the burden on the healthcare systems. Pancreatic stellate cells (PSCs) have been shown to play an important role in the pathogenesis of fibrosis in chronic pancreatitis and pancreatic cancer [3].

In a recent study, we identified a population of stellate cells growing outward of isolated islets which are similar, but not identical to classical PSCs, during the culture of islets from Wistar and Goto-Kakizaki (GK) rats. These cells are named islet stellate cells (ISCs) $[4,5]$, which have been verified to play important roles in islet fibrosis that promotes T2DM progression.

In normal conditions, PSCs stay quiescent and contain vitamin A-storing lipid droplets in their cytoplasm. When suffering from chronic inflammation or oxidative stress, PSCs will be activated and turned into myofibroblast cells, losing their vitamin A-storing lipid droplets, expressing $\alpha$ SMA, ECM components, vimentin, and GFAP, producing cytokines and chemokines such as IL6, IL8, and monocyte chemoattractant protein- (MCP-) 1, as well as having higher proliferation and migration activities. Many recent studies have also shown that there are $\alpha$-SMA-positive 


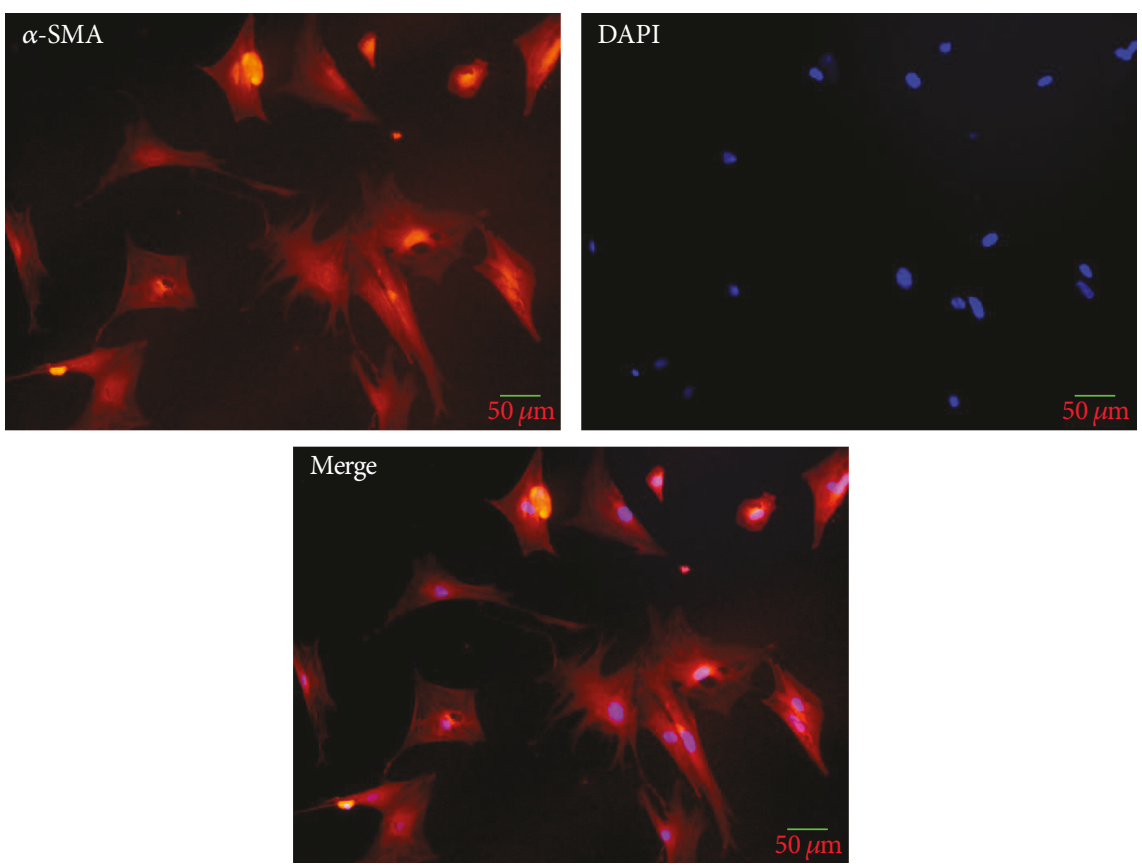

FIGURE 1: A random view of immunofluorescence visualization of $\alpha$-SMA, a marker for ISCs, was performed. All of the ISCs were $\alpha$-SMApositive cell.

cells, identified as PSCs, in the models of islet fibrosis both in humans and animals with T2DM [6-9].

In the previous study, we compared Wistar rat's PSCs with ISCs and found that ISCs contained fewer vitamin A-storing lipid droplets and were more rapidly activated than PSCs in vitro. Activated ISCs express $\alpha$-SMA, ECM components, vimentin, and GFAP, which is similar to activated PSCs. But ISCs have lower rates of proliferation and migration than PSCs in vitro, suggesting that ISCs are similar but not identical to PSCs in morphology and phenotype. In this study, we performed RNA-seq and real-time PCR validation on cultured Wistar rat's PSCs and ISCs to determine the gene differences and splicing variations and provided a global transcriptome comparison between ISCs and PSCs.

\section{Materials and Methods}

2.1. Animals. Three 10-week-old healthy male Wistar rats weighted 300-350 g were chosen for this study, yielding 3 biological duplicates. After measuring fasting blood glucose, the rats were given anesthesia and sacrificed to collect their pancreas. These rats were numbered $A, B$, and $C$, and PSCs from $\mathrm{A}, \mathrm{B}$, and $\mathrm{C}$ were named $\mathrm{A} 1, \mathrm{~B} 1$, and $\mathrm{C} 1$, respectively. Similarly, the ISCs were named A12, B12, and $\mathrm{C} 12$, respectively. Housing and animal experiments were approved by Southeast University Animal Care and Use Committee according to institutional guidelines and national animal welfare.

2.2. Isolation and Culture of ISCs. Islets were isolated from pancreas as previously described [10]. Briefly, pancreas tissues were digested with collagenase $\mathrm{V}(1 \mathrm{mg} / \mathrm{mL}, w / v)$
(Sigma, St. Louis, MO, USA) at $37^{\circ} \mathrm{C}$ for 15 to $18 \mathrm{~min}$. Islets were purified by handpicking twice under a stereomicroscope. Then, islets were precultured in RPMI-1640 supplemented with L-glutamine containing 10\% $(v / v)$ fetal bovine serum (FBS) (Invitrogen, Carlsbad, CA, USA) overnight, followed by handpicking.

After $48 \mathrm{~h}$ in culture, ISCs began to grow out of Wistar islets. Five days later, cells were subcultured in DMEM/ Ham's F12 $(1: 1, v / v)$ containing 10\% FBS. Then, cells at passages 3 were used for experiments.

2.3. Isolation and Culture of PSCs. PSCs were isolated from Wistar rats as described previously [11]. PSCs were cultured in Dulbecco's modified Eagle's medium (DMEM)/ Ham's F12 $(1: 1, v / v)$ containing $10 \%$ fetal bovine serum (FBS) (Invitrogen, Carlsbad, USA). The cells were cultured at the same condition with ISCs. Cells at passages 3 were used for experiments.

2.4. Identification of ISCs via Immunofluorescent Assay. Cells were fixed in $4 \%$ paraformaldehyde in PBS for $20 \mathrm{~min}$ at room temperature. Immunofluorescent staining of stellate cells for $\alpha$-SMA was performed. Cells were incubated overnight at $4^{\circ} \mathrm{C}$ with primary antibody (Abcam, 1:200), followed by a $1 \mathrm{~h}$ treatment with secondary antibody (Jackson ImmunoResearch Laboratories, 1:100). The sections in negative control group were incubated with PBS, instead of primary antibody. And the results indicated that the antibody and the staining worked well. Morphometric analyses were performed using Image J software.

2.5. mRNA Library Construction and Sequencing. Using the Trizol reagent (Invitrogen, CA, USA) according to the 
TABLE 1: Comparisons between clean data and Rattus genome sequences.

\begin{tabular}{|c|c|c|c|c|c|c|}
\hline Mapped statistics & A1 & $\mathrm{B} 1$ & $\mathrm{C} 1$ & A12 & $\mathrm{B} 12$ & $\mathrm{C} 12$ \\
\hline Total reads & $\begin{array}{c}48,691,180 \\
(100 \%)\end{array}$ & $\begin{array}{c}53,917,892 \\
(100 \%)\end{array}$ & $\begin{array}{c}51,532,204 \\
(100 \%)\end{array}$ & $\begin{array}{c}50,777,686 \\
(100 \%)\end{array}$ & $\begin{array}{c}53,749,152 \\
(100 \%)\end{array}$ & $\begin{array}{c}54,536,444 \\
(100 \%)\end{array}$ \\
\hline Total mapped & $\begin{array}{c}44,505,452 \\
(91.4 \%)\end{array}$ & $\begin{array}{c}49,095,515 \\
(91.06 \%)\end{array}$ & $\begin{array}{c}46,914,556 \\
(91.04 \%)\end{array}$ & $\begin{array}{c}46,188,430 \\
(90.96 \%)\end{array}$ & $\begin{array}{c}49,072,733 \\
(91.3 \%)\end{array}$ & $\begin{array}{c}49,552,463 \\
(90.86 \%)\end{array}$ \\
\hline Multiple mapped & $\begin{array}{c}3,128,881 \\
(6.43 \%)\end{array}$ & $\begin{array}{c}3,429,714 \\
(6.36 \%)\end{array}$ & $\begin{array}{c}3,288,274 \\
(6.38 \%)\end{array}$ & $\begin{array}{c}3,172,290 \\
(6.25 \%)\end{array}$ & $\begin{array}{c}3,288,591 \\
(6.12 \%)\end{array}$ & $\begin{array}{c}3,290,626 \\
(6.03 \%)\end{array}$ \\
\hline Uniquely mapped & $\begin{array}{c}41,376,571 \\
(84.98 \%) \\
\end{array}$ & $\begin{array}{c}45,665,801 \\
(84.7 \%)\end{array}$ & $\begin{array}{c}43,626,282 \\
(84.66 \%)\end{array}$ & $\begin{array}{c}43,016,140 \\
(84.71 \%)\end{array}$ & $\begin{array}{c}45,784,142 \\
(85.18 \%)\end{array}$ & $\begin{array}{c}46,261,837 \\
(84.83 \%)\end{array}$ \\
\hline
\end{tabular}

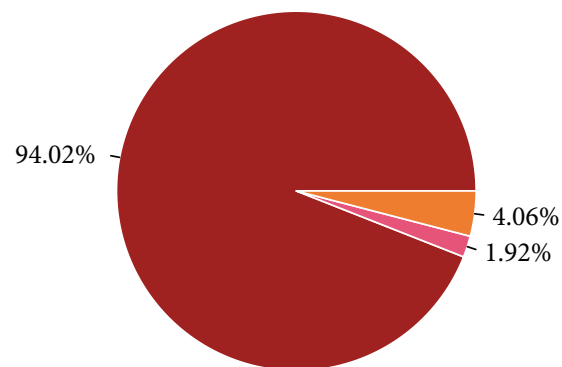

A1

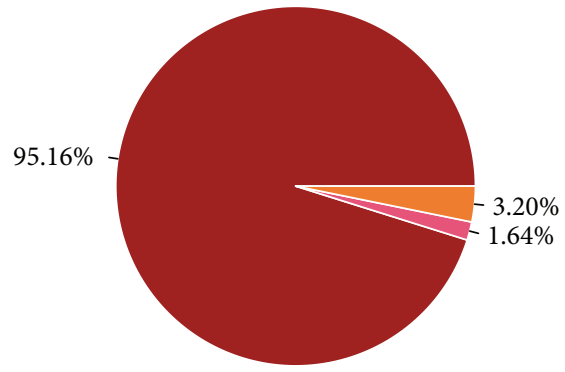

A12

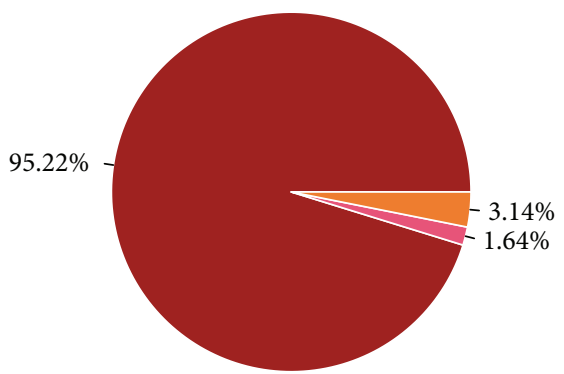

B1

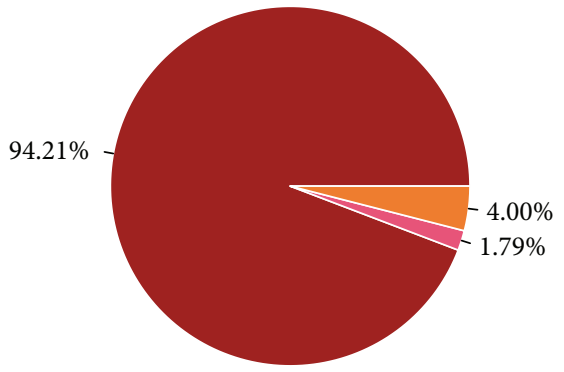

$\mathrm{B} 12$

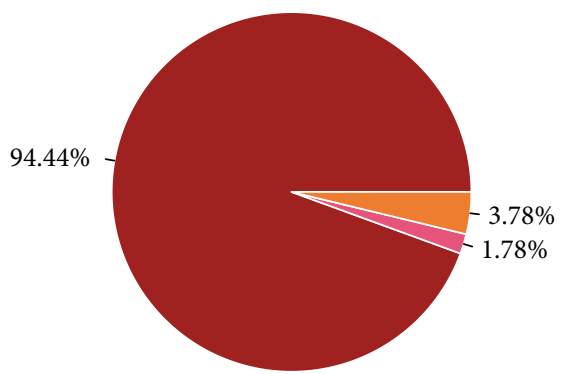

$\mathrm{C} 1$

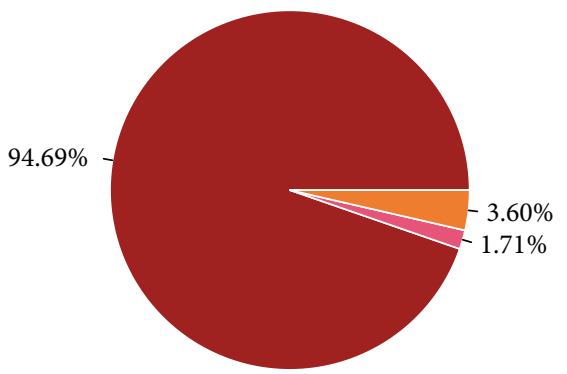

$\mathrm{C} 12$

Exonic

Intergenic

Intronic

FIGURE 2: Mapped read distribution of each read.

manufacturer's protocol, the total RNA was extracted. The purity and quantity of the total RNA were detected using RNA 6000 Nano LabChip Kit (Agilent, CA, USA) and Bio analyzer 2200 with RIN more than 7.0. Then, approximately $2 \mu \mathrm{g}$ of total RNA were subjected to isolate the PolyA mRNA using polyT oligo-attached magnetic beads (Invitrogen). After purification, the mRNA was firstly fragmented into small pieces and then reverse-transcribed to create the final cDNA library based on the protocol of the mRNA-Seq sample preparation kit (Illumina, San Diego, USA). The average insert size for the paired-end libraries was about $300 \mathrm{bps}$. We then performed the paired-end sequencing (100 bps) using the Hiseq3000 platform.

2.6. Functional Enrichment Analysis. The sequenced raw data were filtered to remove low-quality tags such as reads with unknown nucleotides "N," empty reads, and reads with only one copy number. Then, we matched the clean reads to the sequences in the Rattus genome database by Tophat (version 2.0.4) allowing up to two base mismatches. The mapped clean reads were regarded as precise clean reads. For two-factor analysis of variance, we calculated and normalized the number of unambiguous clean reads for each gene to log counts per million using the limma package in $\mathrm{R}$ program.

All detected genes were used for the gene ontology (GO) and Kyoto encyclopedia of genes and genomes (KEGG) enrichment analyses. For the GO analysis, a corrected $P$ value of $<0.05$ was considered as the threshold to determine significant enrichment of the gene sets. Similar as $\mathrm{GO}$ analysis, a $Q$ value $\leq 0.05$ was considered as the threshold to determine significant enrichment of the gene sets for KEGG enrichment analysis.

2.7. Quantitative RT-PCR. Total RNA from the two types of cells were isolated using a rapid extraction method 
(TRI-Reagent, Invitrogen). Real-time PCR was performed on cDNA samples using the FastStart Universal SYBR Green Master (Roche) on Step One Plus system (Applied Biosystems, Foster City, CA, USA). Primers are described in Table S1. The PCR settings used included denaturation $\left(95^{\circ} \mathrm{C}\right.$ for $2 \mathrm{~min}$ ) and amplification steps repeated 40 times $\left(95^{\circ} \mathrm{C}\right.$ for $15 \mathrm{~s}, 55^{\circ} \mathrm{C}$ for $30 \mathrm{~s}, 72^{\circ} \mathrm{C}$ for $30 \mathrm{~s}$, and acquisition temperature for $15 \mathrm{~s}$ ). Analysis was conducted using the sequence detection software supplied. For each sample, the delta delta cycle of threshold (ddCt) (crossing point) values were calculated as the $\mathrm{Ct}$ of the target gene minus the $\mathrm{Ct}$ of the GAPDH gene, assuming PCR efficiency equals to 1 . Gene expression was derived according to the equation $2^{-\mathrm{ddCt}}$, and changes in gene expression were expressed relative to levels of the other group.

\section{Results}

3.1. The Evaluation of the Cells' Purity. The immunofluorescence visualization markers for PSCs are known to include $\alpha$ SMA, vimentin, and GFAP. In the previous study, we demonstrated that these markers were also positive in population of ISCs [4]. To identify the purity of the ISCs isolated from islets of the Wistar rats, we used the $\alpha$-SMA staining to confirm that the cells were the right ones we want to analyze by sequencing. As shown in Figure 1, all the cells were positive for $\alpha$-SMA in a random view, indicating that the purity of ISCs was fair.

3.2. Evaluation of Gene Expression Profiles. To verify the sequencing data coverage area and the depth of coverage, Tophat version 2.0.4 was used for analysis. Total numbers of reads generated from each sample ranged from $48,691,180$ to $54,536,444$. The majority of reads (between $84.66 \%$ and $85.18 \%$ ) were uniquely mapped to the reference Rattus genome sequences across all samples (Table 1). Then, we made statistical analysis on the mapped read distribution from each sample (Figure 2). As shown in Figure 2, the percentage of exonic distribution was ranged from $94.02 \%$ to $95.16 \%$. And the intronic distribution was varied from $3.14 \%$ to $4.06 \%$. The remaining area was intergenic distributed because of the incompletion of the genetic annotation, which may lead to the detection of new genes or new lncRNA. The total number of detected gene from all the samples was 21,901. Specifically, the number of detected genes from each sample was (A1: 18,603, B1: 18,395, C1: 18,305, A12: 17,984, B12: 18,401, and C12: 18,304).

3.3. Analysis of Differentially Expressed Genes. To determine the differentially expressed genes (DEG) between PSC and ISC specimens, negative binomial distribution test (NB) was performed. After filtering differentially expressed genes with FDR-adjusted (FDR false discovery rate) $P$ value $<0.05$ and fold change $>2$, there were 32 significant differentially expressed genes between PSCs and ISCs. Among them, 14 genes were upregulated and 18 were downregulated in Wistar rats' ISCs (Table 2). The obtained gene expression profiles were visualized in a heatmap and a volcano figure (Figure 3).
TABLE 2: The significant differentially expressed genes between PSCs and ISCs.

\begin{tabular}{|c|c|c|}
\hline Genes & Fold change & $Q$ value \\
\hline \multicolumn{3}{|c|}{$\begin{array}{l}\text { Upregulated genes in Wistar rats' } \\
\text { ISCs }\end{array}$} \\
\hline Rpl391 & 77.34 & 0.014 \\
\hline $\operatorname{Bin} 2$ & 26.93 & 0.01 \\
\hline LOC102546539 & 7.10 & $1.00 E-21$ \\
\hline Plscr2 & 3.76 & $8.13 E-15$ \\
\hline Msc & 3.49 & 0.01 \\
\hline Snx 20 & 2.99 & 0.00 \\
\hline Col11a1 & 2.91 & $2.75 E-21$ \\
\hline LOC102550510 & 2.84 & 0.00 \\
\hline Card10 & 2.59 & 0.03 \\
\hline Ldb2 & 2.32 & $2.65 E-05$ \\
\hline Gpr39 & 2.23 & 0.00 \\
\hline RGD1560248 & 2.13 & $2.75 E-07$ \\
\hline Irf8 & 2.09 & $8.20 E-05$ \\
\hline Smoc2 & 2.03 & 0.03 \\
\hline \multicolumn{3}{|c|}{$\begin{array}{l}\text { Downregulated genes in Wistar rats' } \\
\text { ISCs }\end{array}$} \\
\hline Rnf150 & 0.48 & 0.01 \\
\hline Adamts 14 & 0.48 & 0.03 \\
\hline Arrb1 & 0.46 & 0.01 \\
\hline Spon2 & 0.44 & $5.93 E-10$ \\
\hline Fibin & 0.42 & 0.03 \\
\hline Ablim 1 & 0.41 & 0.00 \\
\hline LOC100910790 & 0.39 & $3.53 E-05$ \\
\hline Acan & 0.38 & 0.04 \\
\hline Slc9a2 & 0.37 & 0.00 \\
\hline Actg2 & 0.32 & 0.03 \\
\hline Cdo1 & 0.32 & 0.00 \\
\hline Tnnc1 & 0.31 & 0.01 \\
\hline Plekhh2 & 0.29 & $5.72 E-07$ \\
\hline $\mathrm{Ebf} 2$ & 0.27 & 0.00 \\
\hline Gja5 & 0.23 & $7.12 E-17$ \\
\hline Tbx18 & 0.228723292 & $1.15 E-16$ \\
\hline Prex2 & 0.203772172 & 0.002737756 \\
\hline Gsc & 0 & 0.000404614 \\
\hline
\end{tabular}

3.4. The Differential Gene's KEGG Biological Pathway Enrichment Analysis. The biological pathway analysis was referred to Kyoto encyclopedia of genes and genomes (KEGG) database (http://www.genome.jp/). The top 3 most enriched pathways were taurine and hypotaurine metabolism, cysteine and methionine metabolism, and phototransduction $(Q$ value $<0.05)$ (Table 3$)$. Cdo1 was both involved in taurine and hypotaurine metabolism and cysteine and methionine metabolism, while Arrb1 was involved in phototransduction pathways.

3.5. The Gene Ontology Functional Enrichment Analysis of $D E G s$. To explore the biological role of the genes modulated 


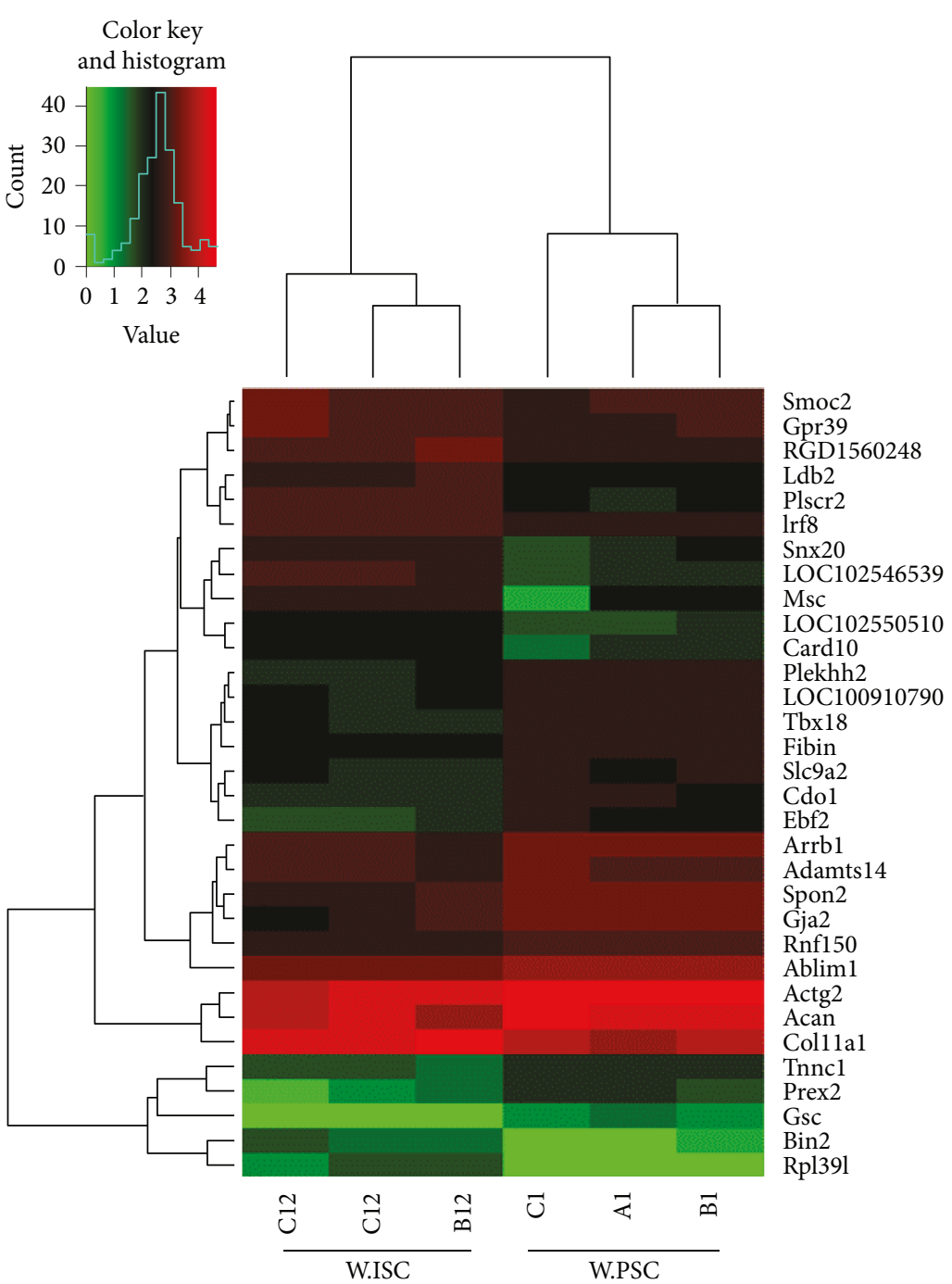

(a)

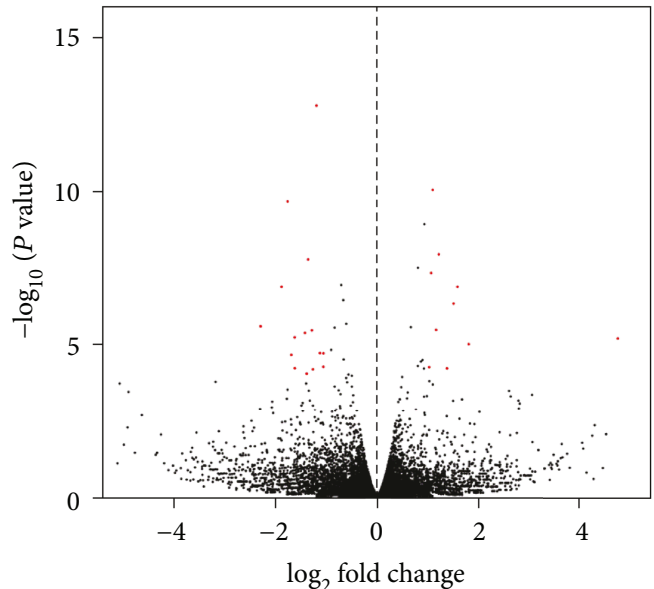

(b)

FIGURE 3: Heatmap and volcano plots showing DEGs between Wistar rats' PSCs and ISCs. Heatmap shows the hierarchically clustered genes (a). Upregulated levels of gene expression are displayed as red bars while downregulated levels are displayed as green bars. Volcano plot shows the overall distribution of DEGs (b). Genes with fold change $>2$ and statistical significance are marked with red dots.

TABLE 3: KEGG pathway analysis of DEGs in Wistar rats' PSCs and ISCs.

\begin{tabular}{lccrr}
\hline Term & ID & Q value & Gene ID & Gene \\
\hline Taurine and hypotaurine metabolism & rno00430 & 0.008177 & 81,718 & Cdo1 \\
Phototransduction & rno04744 & 0.025229 & 25,387 & Arrb1 \\
Cysteine and methionine metabolism & rno00270 & 0.036734 & 81,718 & Cdo1 \\
\hline
\end{tabular}

in PSCs and ISCs at the transcriptional level, we made function annotation for each DGEs through gene ontology (GO) functional enrichment analysis.

The top 10 categories of each part of GO analysis that were significantly enriched with a $Q$ value $<0.05$ are shown in Figure 4.

3.6. Validation of $m R N A-S e q$ Results. We confirmed the expression level of 10 DEGs ( 5 upregulated and 5 downregulated genes) using real-time PCR (Figure 5). The genes which we validated should meet the following principles: firstly, the fold change of DEGs was as large as possible. Secondly, the gene expression, at transcription level, was enough to be detected via real-time PCR. Thirdly, the genes were associated with different biological features between Wistar rats' PSCs and ISCs, such as proliferation, migration, and apoptosis.

The results of the validation via real-time PCR showed that, although the RQ was different from the fold change, the tendency was in consistent with the results presented in the transcriptional analysis. 


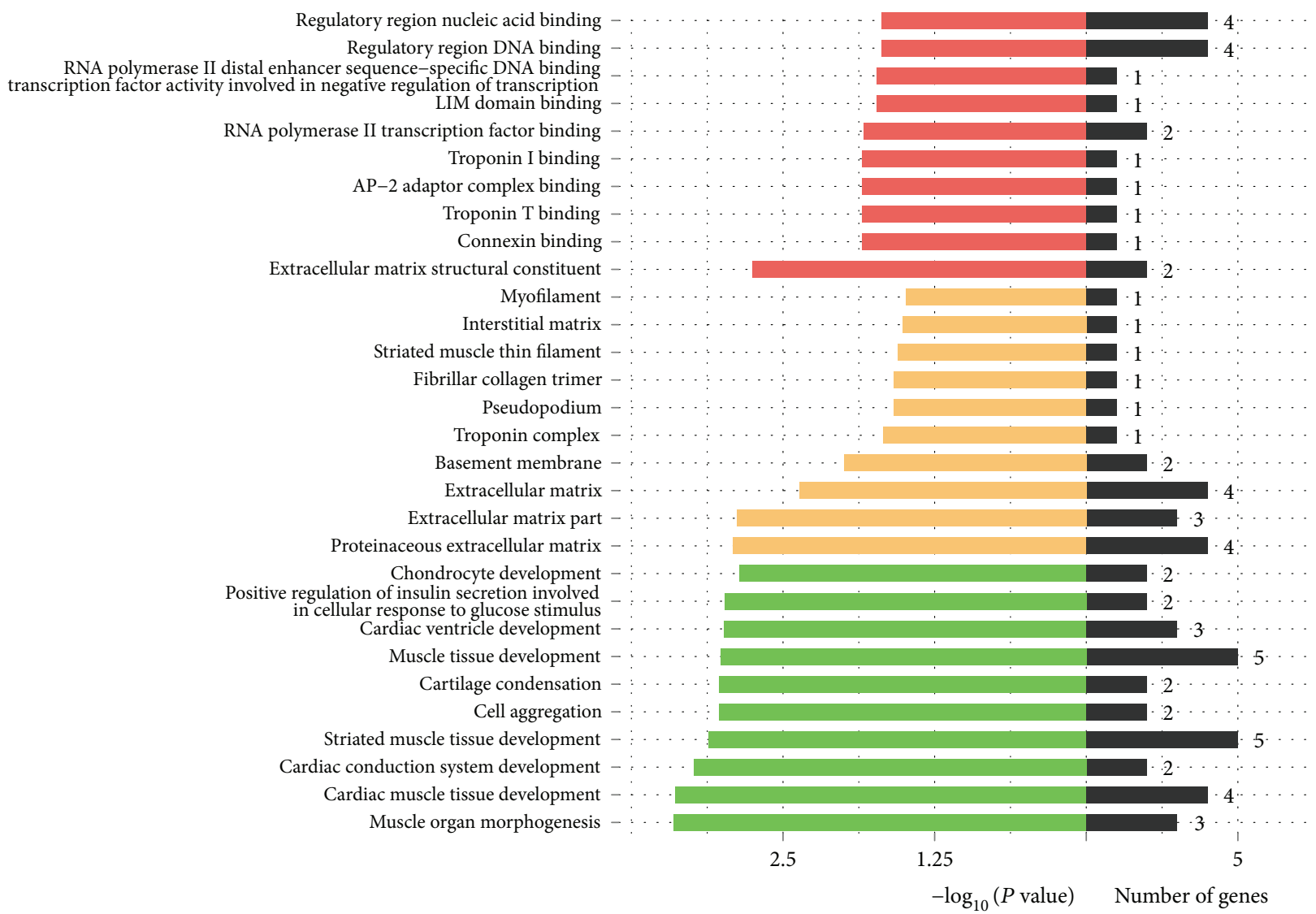

FIGURE 4: DEGs' GO enrichment classification. Red bars represent the molecular function. Yellow bars represent the cellular component while the green represents the biological process.

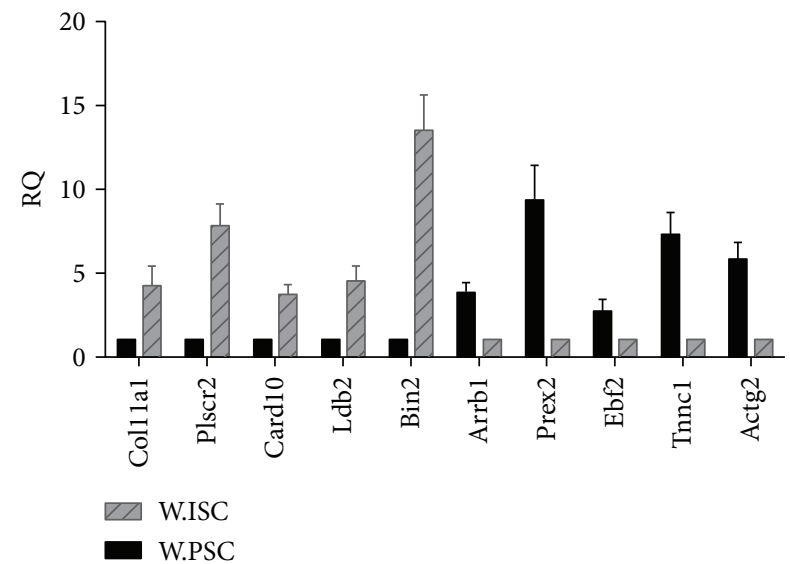

FIGURE 5: Validation of mRNA-seq to validate the 10 differential expression genes by real-time PCR. Black bars represent the gene expression levels of Wistar rats' PSCs while gray bars represent the relative gene expression levels of Wistar rats' ISCs.

\section{Discussion}

There is the evidence that human fibroblasts exhibit topographic differentiation via genome-wide expression profiling [12]. Fibroblasts from each site displayed distinct and characteristic transcriptional patterns, suggesting that fibroblasts at different locations in the body should be considered distinctly differentiated cell types. The topographic differentiation of fibroblasts results in different biologic specificity. A recent study using immunohistochemistry assay confirmed that there exist $\alpha$-SMA-positive cells in the fibrosis area of islets, which are recognized to be involved in the progression of islet fibrosis [13]. It seems likely that such myofibroblastlike cells in islets should be different from PSCs, since the latter cells are located in the exocrine glands of the pancreas.

Our study showed that there was a difference in transcription levels of Wistar rats' PSCs and ISCs, and there existed 32 differentially expressed genes, accounting for $0.1461 \%$ of all the 21,901 genes detected. The mRNA levels of these genes were further confirmed by real-time PCR. The results of the RNA-seq confirmed our hypothesis that ISCs are similar but not identical to PSCs. In this experiment, we focused on the difference at the transcription level between these two types of fibroblasts. So we think that ISCs and PSCs have the same origin but from different locations. PSCs play a part in pancreatic exocrine glands, while ISCs play a part in islets due to their location.

Our data also provided valuable information regarding the subtle but important differences of islet stellate cells versus pancreatic stellate cells. We showed that COL11A1 expression was significantly upregulated in Wistar rats' ISCs, with a fold change of 2.91 . 
Minor fibrillar collagens, type V and type XI, were considered to act as nucleators, controlling the assembly of collagen fibrils and participating in proper type II collagen fibril formation [14]. COL11A1 encodes one of the two chains of collagen type XI, $\alpha 1$ chain [15]. Col11A1 is highly expressed by activated stromal cells in multiple cancers which are mostly invasive, such as oral cavity/pharynx, head and neck, breast, lung, esophagus, stomach, colon, and ovary [16-26]. Recent research has emphasized the role of colllal in various cancers. Coll11a1 may play roles in metastasis, angiogenesis, and drug resistance, as well as its potential value in screening tests and as a therapeutic target [27]. One study shows that proCOL11A1 presents a strong immunohistochemical staining within the stroma cells/cancer-associated fibroblasts (CAFs) of pancreatic ductal adenocarcinomas (PDAC), but not strongly costaining with chronic pancreatitis [21]. ProCOL11A1-positive cells presented costaining mesenchymal, stellate, and epithelial markers such as vimentin, $\alpha$-SMA, or desmin in different proportions, suggesting that proCOL11A1-positive cells might be involved in epithelial-mesenchymal transition (EMT).

In agreement with our previous experimental results, this study showed that ISCs contain fewer vitamin Astoring lipid droplets and are more rapidly activated than PSCs in vitro. Activated ISCs express abundant quantity of $\alpha$-SMA and ECM components that may lead to fibrosis. Taking the location of the ISCs into consideration, the activated ISCs are the most likely causes of islet fibrosis in the condition of diabetes. Given our result of mRNA sequencing and considering previous findings, the upregulated expression gene of Coll11a1 in Wistar rats' ISCs is likely to be a key gene underlying the differential pathophysiology of ISCs and PSCs.

In conclusion, our data showed the differences of ISCs and PSCs at the transcriptional level. Genes like Coll11a1 may be a key to differences in pathophysiology of ISCs and PSCs. Identification of the gene expression profiles would enrich our current understanding of the ISCs, which also confirmed the previous assumption that ISC and PSC are similar but not identical in morphology and phenotype. In our previous study, comparing with normal status, ISCs in diabetic status showed a significantly greater migration, a larger rate of apoptosis and viability, and a higher level of ECM component secretion [28]. Provided that there existed a molecular switch that can be used to reverse this phenomenon and subsequently relieve the islet fibrosis in terminal stage of diabetes, this could be a cue to develop novel efficiency therapy approaches for diabetes.

\section{Conflicts of Interest}

The authors declare that there is no conflict of interests regarding the publication of this paper.

\section{Acknowledgments}

This study was supported by the National Nature Science Foundation of China (NSFC-81354867).

\section{Supplementary Materials}

Table S1: the primers of validated genes. (Supplementary Materials)

\section{References}

[1] N. A. Sherry, E. B. Tsai, and K. C. Herold, "Natural history of beta-cell function in type 1 diabetes," Diabetes, vol. 54, Supplement 2, pp. S32-S39, 2005.

[2] J. L. Chiasson and R. Rabasa-Lhoret, "Prevention of type 2 diabetes: insulin resistance and beta-cell function," Diabetes, vol. 53, Supplement 3, pp. S34-S38, 2004.

[3] M. Apte, R. Pirola, and J. Wilson, "The fibrosis of chronic pancreatitis: new insights into the role of pancreatic stellate cells," Antioxidants \& Redox Signaling, vol. 15, no. 10, pp. 2711-2722, 2011.

[4] M. Zha, F. Li, W. Xu, B. Chen, and Z. Sun, "Isolation and characterization of islet stellate cells in rat," Islets, vol. 6, no. 2, article e28701, 2014.

[5] F. F. Li, B. J. Chen, W. Li et al., "Islet stellate cells isolated from fibrotic islet of Goto-Kakizaki rats affect biological behavior of beta-cell," Journal of diabetes research, vol. 2016, Article ID 6924593, 9 pages, 2016.

[6] E. Lee, G. R. Ryu, S. H. Ko et al., “Antioxidant treatment may protect pancreatic beta cells through the attenuation of islet fibrosis in an animal model of type 2 diabetes," Biochemical and Biophysical Research Communications, vol. 414, no. 2, pp. 397-402, 2011.

[7] R. Saito, S. Yamada, Y. Yamamoto et al., "Conophylline suppresses pancreatic stellate cells and improves islet fibrosis in Goto-Kakizaki rats," Endocrinology, vol. 153, no. 2, pp. 621-630, 2012.

[8] S. P. Datar and R. R. Bhonde, "Islet-derived stellate-like cells as a novel source for islet neogenesis in chicks," Poultry Science, vol. 88, no. 3, pp. 654-660, 2009.

[9] J. Yang, R. T. Waldron, S. HY et al., "Insulin promotes proliferation and fibrosing responses in activated pancreatic stellate cells," American journal of physiology Gastrointestinal and liver physiology, vol. 311, no. 4, pp. G675-G687, 2016.

[10] M. Zha, M. Zhang, S. Shan et al., "Effects of islet neogenesisassociated protein pentadecapeptide on cell mass and insulin secretion of pancreatic beta-cells," Journal of Endocrinological Investigation, vol. 35, no. 7, pp. 634-639, 2012.

[11] M. G. Bachem, E. Schneider, H. Gross et al., "Identification, culture, and characterization of pancreatic stellate cells in rats and humans," Gastroenterology, vol. 115, no. 2, pp. 421-432, 1998.

[12] H. Y. Chang, J. T. Chi, S. Dudoit et al., "Diversity, topographic differentiation, and positional memory in human fibroblasts," Proceedings of the National Academy of Sciences of the United States of America, vol. 99, no. 20, pp. 12877-12882, 2002.

[13] E. Lee, G. R. Ryu, S.-H. Ko, Y.-B. Ahn, and K.-H. Song, "A role of pancreatic stellate cells in islet fibrosis and $\beta$ cell dysfunction in type 2 diabetes mellitus," Biochemical and Biophysical Research Communications, vol. 485, no. 2, pp. 328-334, 2017.

[14] K. E. Kadler, A. Hill, and E. G. Canty-Laird, "Collagen fibrillogenesis: fibronectin, integrins, and minor collagens as organizers and nucleators," Current Opinion in Cell Biology, vol. 20, no. 5, pp. 495-501, 2008. 
[15] F. Mio, K. Chiba, Y. Hirose et al., "A functional polymorphism in COL11A1, which encodes the a 1 chain of type XI collagen, is associated with susceptibility to lumbar disc herniation," American Journal of Human Genetics, vol. 81, no. 6, pp. 1271-1277, 2007.

[16] S. Pavlides, A. Tsirigos, I. Vera et al., "Transcriptional evidence for the "reverse Warburg effect" in human breast cancer tumor stroma and metastasis: similarities with oxidative stress, inflammation, Alzheimer's disease, and "neuron-glia metabolic coupling"," Aging, vol. 2, no. 4, pp. 185-199, 2010.

[17] A. Planche, M. Bacac, P. Provero et al., "Identification of prognostic molecular features in the reactive stroma of human breast and prostate cancer," PLoS One, vol. 6, no. 5, article e18640, 2011.

[18] C. E. Schmalbach, D. B. Chepeha, T. J. Giordano et al., "Molecular profiling and the identification of genes associated with metastatic oral cavity/pharynx squamous cell carcinoma," Archives of Otolaryngology-Head \& Neck Surgery, vol. 130, no. 3, pp. 295-302, 2004.

[19] J. C. Sok, M. A. Kuriakose, V. B. Mahajan, A. N. Pearlman, M. D. DeLacure, and F. A. Chen, "Tissue-specific gene expression of head and neck squamous cell carcinoma in vivo by complementary DNA microarray analysis," Archives of Otolaryngology-Head \& Neck Surgery, vol. 129, no. 7, pp. 760-770, 2003.

[20] J. C. Sok, J. A. Lee, S. Dasari et al., "Collagen type XI a1 facilitates head and neck squamous cell cancer growth and invasion," British Journal of Cancer, vol. 109, no. 12, pp. 3049-3056, 2013.

[21] C. Garcia-Pravia, J. A. Galvan, N. Gutierrez-Corral et al., "Overexpression of COL11A1 by cancer-associated fibroblasts: clinical relevance of a stromal marker in pancreatic cancer," PLoS One, vol. 8, no. 10, article e78327, 2013.

[22] K. K. Wang, N. Liu, N. Radulovich et al., "Novel candidate tumor marker genes for lung adenocarcinoma," Oncogene, vol. 21 , no. 49 , pp. 7598-7604, 2002.

[23] X. SH, L. J. Qian, H. Z. Mou et al., "Difference of gene expression profiles between esophageal carcinoma and its pericancerous epithelium by gene chip," World Journal of Gastroenterology, vol. 9, no. 3, pp. 417-422, 2003.

[24] Y. Zhao, T. Zhou, A. Li et al., "A potential role of collagens expression in distinguishing between premalignant and malignant lesions in stomach," The Anatomical Record: Advances in Integrative Anatomy and Evolutionary Biology, vol. 292, no. 5, pp. 692-700, 2009.

[25] J. A. Galvan, J. Garcia-Martinez, F. Vazquez-Villa et al., "Validation of COL11A1/procollagen 11A1 expression in TGF-beta1-activated immortalised human mesenchymal cells and in stromal cells of human colon adenocarcinoma," $B M C$ Cancer, vol. 14, no. 1, p. 867, 2014.

[26] D. J. Cheon, Y. Tong, M. S. Sim et al., “A collagen-remodeling gene signature regulated by TGF- $\beta$ signaling is associated with metastasis and poor survival in serous ovarian cancer," Clinical Cancer Research, vol. 20, no. 3, pp. 711-723, 2014.

[27] Z. Raglow and S. M. Thomas, "Tumor matrix protein collagen XIalpha1 in cancer," Cancer Letters, vol. 357, no. 2, pp. 448453, 2015.

[28] W. Xu, W. Li, Y. Wang et al., "Regenerating islet-derived protein 1 inhibits the activation of islet stellate cells isolated from diabetic mice," Oncotarget, vol. 6, no. 35, pp. 37054-37065, 2015. 


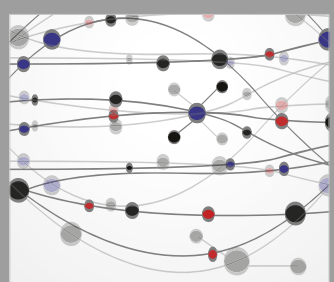

The Scientific World Journal
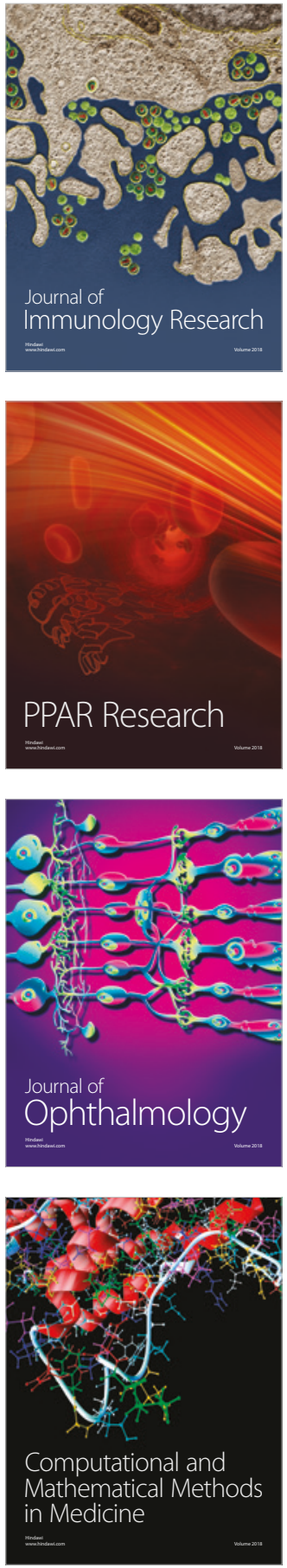



Gastroenterology Research and Practice

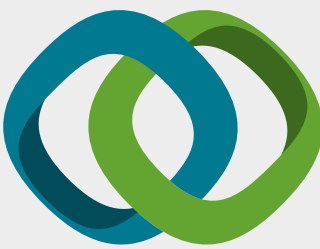

\section{Hindawi}

Submit your manuscripts at

www.hindawi.com
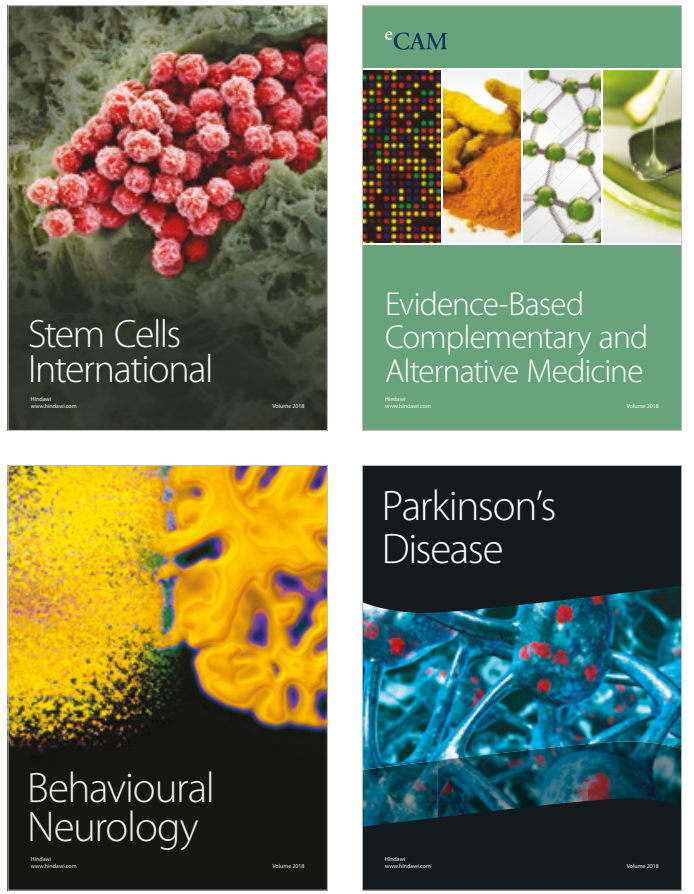

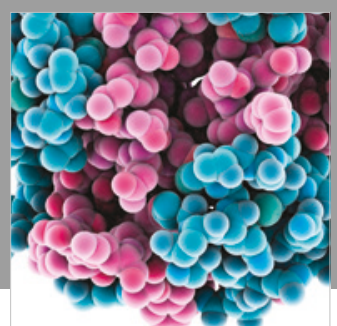

ournal of

Diabetes Research

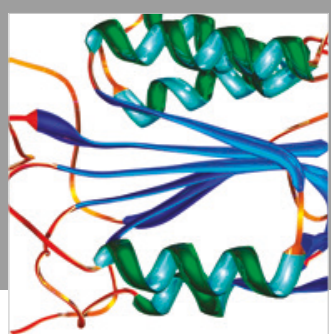

Disease Markers
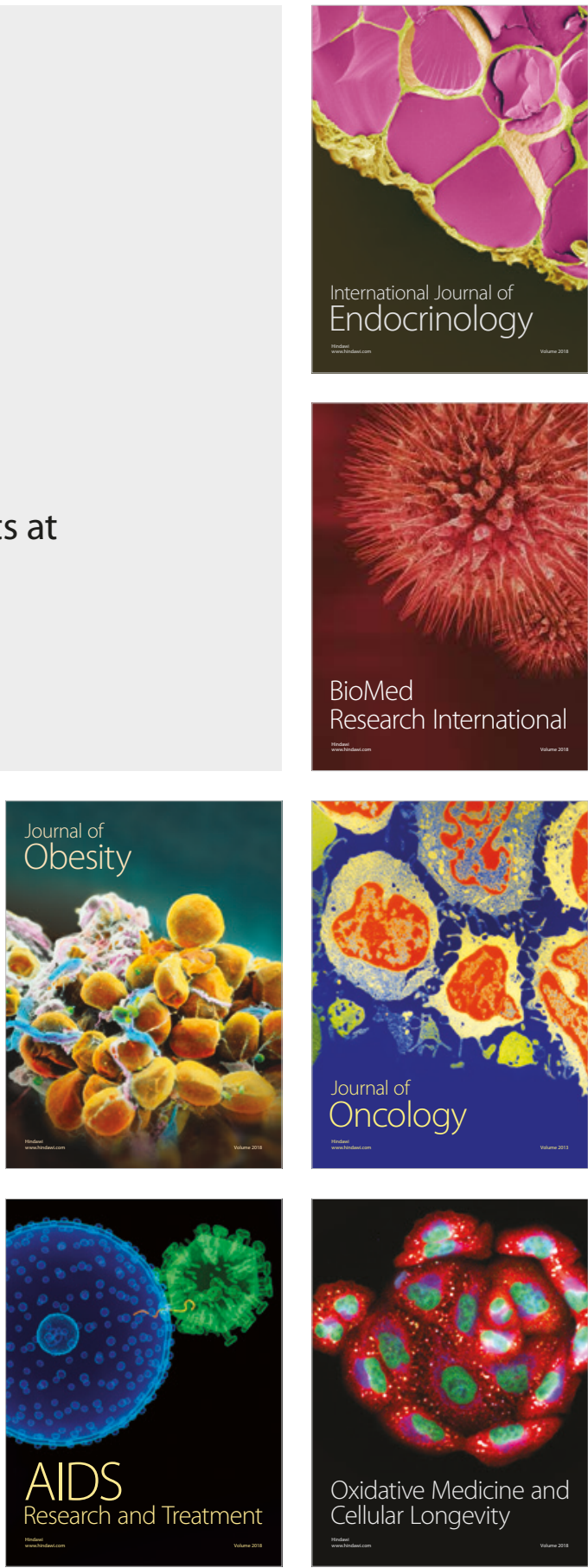